\title{
Ground state solutions of Kirchhoff-type fractional Dirichlet problem with $p$-Laplacian
}

\section{Taiyong Chen ${ }^{*}$ and Wenbin Liu ${ }^{1}$}

\section{"Correspondence:}

taiyongchen@cumt.edu.cn

'School of Mathematics, China

University of Mining and

Technology, Xuzhou, P.R. China

\begin{abstract}
We consider the Kirchhoff-type $p$-Laplacian Dirichlet problem containing the left and right fractional derivative operators. By using the Nehari method in critical point theory, we obtain the existence theorem of ground state solutions for such Dirichlet problem.
\end{abstract}

MSC: $26 \mathrm{~A} 33 ; 34 \mathrm{~B} 15 ; 58 \mathrm{E} 05$

Keywords: Kirchhoff-type equation; Fractional p-Laplacian; Dirichlet problem; Ground state solution; Nehari manifold

\section{Introduction}

In the present paper, we discuss the existence of ground state solutions for the Kirchhofftype fractional Dirichlet problem with $p$-Laplacian of the form

$$
\left\{\begin{array}{l}
\left(a+\left.\left.b \int_{0}^{T}\right|_{0} D_{t}^{\alpha} u(t)\right|^{p} d t\right)^{p-1}{ }_{t} D_{T}^{\alpha} \phi_{p}\left({ }_{0} D_{t}^{\alpha} u(t)\right)=f(t, u(t)), \quad t \in(0, T), \\
u(0)=u(T)=0
\end{array}\right.
$$

where $a, b>0, p>1$ are constants, ${ }_{0} D_{t}^{\alpha}$ and ${ }_{t} D_{T}^{\alpha}$ are the left and right Riemann-Liouville fractional derivatives of order $\alpha \in(1 / p, 1]$, respectively, $\phi_{p}: \mathbb{R} \rightarrow \mathbb{R}$ is the $p$-Laplacian defined by

$$
\phi_{p}(s)=|s|^{p-2} s \quad(s \neq 0), \quad \phi_{p}(0)=0,
$$

and $f \in C^{1}([0, T] \times \mathbb{R}, \mathbb{R})$.

The Kirchhoff equation [21] is an extension of the wave equation which comes from the free vibrations of elastic strings and takes into account the changes in length of the string produced by transverse vibrations. In addition, the fractional order models are more appropriate than the integer order models in real world owing to the fact that the fractional derivatives offer a wonderful tool to describe the memory and hereditary properties of a great deal of processes and materials [12, 15, 16, 22, 25]. Moreover, the $p$-Laplacian [23] often appears in non-Newtonian fluid theory, nonlinear elastic mechanics, and so on.

Notice that, when $a=1, b=0$, and $p=2$, the left-hand side of equation of BVP (1), which is nonlinear and nonlocal, reduces to the linear operator ${ }_{t} D_{T 0}^{\alpha} D_{t}^{\alpha}$, and further reduces to the local operator $-d^{2} / d t^{2}$ when $\alpha=1$.

(c) The Author(s) 2018. This article is distributed under the terms of the Creative Commons Attribution 4.0 International License (http://creativecommons.org/licenses/by/4.0/), which permits unrestricted use, distribution, and reproduction in any medium, provided you give appropriate credit to the original author(s) and the source, provide a link to the Creative Commons license, and indicate if changes were made. 
In recent years, there have been many authors to study the fractional boundary value problems (BVPs for short) $[1,3,4,7,11,17]$ and the Kirchhoff equations [2, 6, 8, 10, 24, 26], and to obtain numerous important results. In addition, the models containing left and right fractional derivatives have been recently gaining more attention $[5,9,13,14,18,19$, 28 ] because of the applications in physical phenomena exhibiting anomalous diffusion.

Motivated by the above works, in this paper, we discuss the existence of nontrivial ground state solutions for BVP (1). The main tool used here is the Nehari method.

For the nonlinearity $f$, we make the following assumptions throughout this paper.

$\left(\mathrm{H}_{1}\right)$ The mapping $x \rightarrow f(t, x) /|x|^{p^{2}-1}$ is strictly increasing on $\mathbb{R} \backslash\{0\}$ for $\forall t \in[0, T]$.

$\left(\mathrm{H}_{2}\right) f(t, x)=o\left(|x|^{p-1}\right)$ as $|x| \rightarrow 0$ uniformly for $\forall t \in[0, T]$.

$\left(\mathrm{H}_{3}\right)$ There exist two constants $\mu>p^{2}, R>0$ such that

$$
0<\mu F(t, x) \leq x f(t, x), \quad \forall t \in[0, T], x \in \mathbb{R} \text { with }|x| \geq R,
$$

where $F(t, x)=\int_{0}^{x} f(t, s) d s$.

Now we state our main result.

Theorem 1.1 Let $\left(\mathrm{H}_{1}\right)-\left(\mathrm{H}_{3}\right)$ be satisfied. Then BVP (1) possesses at least one nontrivial ground state solution.

The rest of this paper is organized as follows. Some preliminary results are presented in Sect. 2. Section 3 is devoted to proving Theorem 1.1.

\section{Preliminaries}

In this section, we present some basic definitions and notations of the fractional calculus $[20,27]$. Moreover we introduce a fractional Sobolev space and some properties of this space [19].

Definition 2.1 For $\gamma>0$, the left and right Riemann-Liouville fractional integrals of order $\gamma$ of a function $u:[a, b] \rightarrow \mathbb{R}$ are given by

$$
\begin{aligned}
& { }_{a} I_{t}^{\gamma} u(t)=\frac{1}{\Gamma(\gamma)} \int_{a}^{t}(t-s)^{\gamma-1} u(s) d s, \\
& { }_{t} I_{b}^{\gamma} u(t)=\frac{1}{\Gamma(\gamma)} \int_{t}^{b}(s-t)^{\gamma-1} u(s) d s,
\end{aligned}
$$

provided that the right-hand side integrals are pointwise defined on $[a, b]$, where $\Gamma(\cdot)$ is the gamma function.

Definition 2.2 For $n-1 \leq \gamma<n(n \in \mathbb{N})$, the left and right Riemann-Liouville fractional derivatives of order $\gamma$ of a function $u:[a, b] \rightarrow \mathbb{R}$ are given by

$$
\begin{aligned}
& { }_{a} D_{t}^{\gamma} u(t)=\frac{d^{n}}{d t^{n}} a I_{t}^{n-\gamma} u(t), \\
& { }_{t} D_{b}^{\gamma} u(t)=(-1)^{n} \frac{d^{n}}{d t^{n}} t I_{b}^{n-\gamma} u(t) .
\end{aligned}
$$


Remark 2.3 When $\gamma=1$, one can obtain from Definitions 2.1 and 2.2 that

$$
{ }_{a} D_{t}^{1} u(t)=u^{\prime}(t), \quad{ }_{t} D_{b}^{1} u(t)=-u^{\prime}(t)
$$

where $u^{\prime}$ is the usual first-order derivative of $u$.

Definition 2.4 For $0<\alpha \leq 1$ and $1<p<\infty$, the fractional derivative space $E_{0}^{\alpha, p}$ is defined by the closure of $C_{0}^{\infty}((0, T), \mathbb{R})$ with respect to the following norm:

$$
\|u\|_{E^{\alpha, p}}=\left(\|u\|_{L^{p}}^{p}+\left\|D_{0}^{\alpha} u\right\|_{L^{p}}^{p}\right)^{\frac{1}{p}}
$$

where $\|u\|_{L^{p}}=\left(\int_{0}^{T}|u(t)|^{p} d t\right)^{1 / p}$ is the norm of $L^{p}((0, T), \mathbb{R})$.

Remark 2.5 It is obvious that, for $u \in E_{0}^{\alpha, p}$, one has

$$
u,{ }_{0} D_{t}^{\alpha} u \in L^{p}((0, T), \mathbb{R}), \quad u(0)=u(T)=0 .
$$

Lemma 2.6 (see [19]) Let $0<\alpha \leq 1$ and $1<p<\infty$. The fractional derivative space $E_{0}^{\alpha, p}$ is a reflexive and separable Banach space.

Lemma 2.7 (see [19]) Let $0<\alpha \leq 1$ and $1<p<\infty$. For $u \in E_{0}^{\alpha, p}$, one has

$$
\|u\|_{L^{p}} \leq C_{p}\left\|_{0} D_{t}^{\alpha} u\right\|_{L^{p}}
$$

where

$$
C_{p}=\frac{T^{\alpha}}{\Gamma(\alpha+1)}>0
$$

is a constant. Moreover, if $\alpha>1 / p$, then

$$
\|u\|_{\infty} \leq C_{\infty}\left\|_{0} D_{t}^{\alpha} u\right\|_{L^{p}}
$$

where $\|u\|_{\infty}=\max _{t \in[0, T]}|u(t)|$ is the norm of $C([0, T], \mathbb{R})$ and

$$
C_{\infty}=\frac{T^{\alpha-\frac{1}{p}}}{\Gamma(\alpha)(\alpha q-q+1)^{\frac{1}{q}}}>0, \quad q=\frac{p}{p-1}>1
$$

are two constants.

Remark 2.8 By (2), we can consider the space $E_{0}^{\alpha, p}$ with the norm

$$
\|u\|_{E^{\alpha, p}}=\left\|{ }_{0} D_{t}^{\alpha} u\right\|_{L^{p}}
$$

in what follows.

Lemma 2.9 (see [19]) Let $1 / p<\alpha \leq 1$ and $1<p<\infty$. The imbedding of $E_{0}^{\alpha, p}$ in $C([0, T], \mathbb{R})$ is compact. 


\section{Ground state solutions of BVP (1)}

The purpose of this section is to prove our main result via the Nehari method. To this end, we are going to set up the corresponding variational framework of BVP (1).

Define the functional $I: E_{0}^{\alpha, p} \rightarrow \mathbb{R}$ by

$$
\begin{aligned}
I(u) & =\frac{1}{b p^{2}}\left(a+b \int_{0}^{T}\left|{ }_{0} D_{t}^{\alpha} u(t)\right|^{p} d t\right)^{p}-\int{ }_{0}^{T} F(t, u(t)) d t-\frac{a^{p}}{b p^{2}} \\
& =\frac{1}{b p^{2}}\left(a+b\|u\|_{E^{\alpha, p}}^{p}\right)^{p}-\int_{0}^{T} F(t, u(t)) d t-\frac{a^{p}}{b p^{2}} .
\end{aligned}
$$

Then there is one-to-one correspondence between the critical points of energy functional $I$ and the weak solutions of BVP (1). It is easy to check from (3), (4), and $f \in C^{1}([0, T] \times$ $\mathbb{R}, \mathbb{R}$ ) that the functional $I$ is well defined on $E_{0}^{\alpha, p}$ and is second-order continuously Fréchet differentiable, that is, $I \in C^{2}\left(E_{0}^{\alpha, p}, \mathbb{R}\right)$. Furthermore, we have

$$
\begin{aligned}
\left\langle I^{\prime}(u), v\right\rangle= & \left(a+b\|u\|_{E^{\alpha, p}}^{p}\right)^{p-1} \int_{0}^{T} \phi_{p}\left({ }_{0} D_{t}^{\alpha} u(t)\right)_{0} D_{t}^{\alpha} v(t) d t \\
& -\int_{0}^{T} f(t, u(t)) v(t) d t, \quad \forall u, v \in E_{0}^{\alpha, p},
\end{aligned}
$$

which yields

$$
\left\langle I^{\prime}(u), u\right\rangle=\left(a+b\|u\|_{E^{\alpha, p}}^{p}\right)^{p-1}\|u\|_{E^{\alpha, p}}^{p}-\int{ }_{0}^{T} f(t, u(t)) u(t) d t .
$$

Now let us define

$$
\mathcal{N}=\left\{u \in E_{0}^{\alpha, p} \backslash\{0\} \mid G(u)=0\right\}
$$

where

$$
G(u)=\left\langle I^{\prime}(u), u\right\rangle
$$

Thus we know that any non-zero critical point of $I$ must be on $\mathcal{N}$. In the following, for simplicity, let

$$
M_{u}=a+b\|u\|_{E^{\alpha, p}}^{p} .
$$

From $\left(\mathrm{H}_{1}\right)$, one has

$$
f_{2}^{\prime}(t, x) x^{2} \geq\left(p^{2}-1\right) f(t, x) x, \quad \forall(t, x) \in[0, T] \times(\mathbb{R} \backslash\{0\}),
$$

where $f_{2}^{\prime}(t, x)=\frac{\partial f(t, x)}{\partial x}$. Then, for $u \in \mathcal{N}$, we have

$$
\begin{aligned}
\left\langle G^{\prime}(u), u\right\rangle= & b p(p-1) M_{u}^{p-2}\|u\|_{E^{\alpha, p}}^{2 p}+p M_{u}^{p-1}\|u\|_{E^{\alpha, p}}^{p} \\
& -\int_{0}^{T} f_{2}^{\prime}(t, u(t)) u^{2}(t) d t-\int_{0}^{T} f(t, u(t)) u(t) d t
\end{aligned}
$$




$$
\begin{aligned}
& \leq M_{u}^{p-2}\|u\|_{E^{\alpha, p}}^{p}\left(b p^{2}\|u\|_{E^{\alpha, p}}^{p}+a p\right)-p^{2} \int_{0}^{T} f(t, u(t)) u(t) d t \\
& =a\left(p-p^{2}\right) M_{u}^{p-2}\|u\|_{E^{\alpha, p}}^{p}<0,
\end{aligned}
$$

which means that $\mathcal{N}$ has a $C^{1}$ structure and is a manifold.

Lemma 3.1 Assume that $\left(\mathrm{H}_{1}\right)$ holds. If $u \in \mathcal{N}$ is a critical point of $\left.I\right|_{\mathcal{N}}$, then $I^{\prime}(u)=0$, that is, $\mathcal{N}$ is a natural constraint for $I$.

Proof If $u \in \mathcal{N}$ is a critical point of $\left.I\right|_{\mathcal{N}}$, then there exists a Lagrange multiplier $\lambda \in \mathbb{R}$ such that

$$
I^{\prime}(u)=\lambda G^{\prime}(u)
$$

Then we get

$$
\left\langle I^{\prime}(u), u\right\rangle=\lambda\left\langle G^{\prime}(u), u\right\rangle=0,
$$

which together with (6) yields $\lambda=0$. So we have $I^{\prime}(u)=0$.

In order to discuss the critical points of $\left.I\right|_{\mathcal{N}}$, we need to investigate the structure of $\mathcal{N}$.

Lemma 3.2 Assume that $\left(\mathrm{H}_{1}\right)-\left(\mathrm{H}_{3}\right)$ hold. For each $u \in E_{0}^{\alpha, p} \backslash\{0\}$, there is unique $s=s(u) \in$ $\mathbb{R}^{+}$such that $s u \in \mathcal{N}$.

Proof First, we claim that there exist constants $\rho, \sigma>0$ such that

$$
I(u)>0, \quad \forall u \in B_{\rho}(0) \backslash\{0\}, \quad I(u) \geq \sigma, \quad \forall u \in \partial B_{\rho}(0),
$$

where $B_{\rho}(0)$ is an open ball in $E_{0}^{\alpha, p}$ with the radius $\rho$ and centered at 0 , and $\partial B_{\rho}(0)$ denotes its boundary. That is, by $I(0)=0,0$ is a strict local minimizer of $I$. In fact, from $\left(\mathrm{H}_{2}\right)$, there are two constants $0<\varepsilon<1, \delta>0$ such that

$$
F(t, x) \leq \frac{(1-\varepsilon) a^{p-1}}{p C_{p}^{p}}|x|^{p}, \quad \forall(t, x) \in[0, T] \times[-\delta, \delta]
$$

where $C_{p}>0$ is a constant defined in (2). Let $\rho=\delta / C_{\infty}$ and $\sigma=\varepsilon a^{p-1} \rho^{p} / p$, where $C_{\infty}>0$ is a constant defined in (3). Then, by (3) and (4), one has

$$
\|u\|_{\infty} \leq C_{\infty}\|u\|_{E^{\alpha, p}} \leq \delta, \quad \forall u \in \overline{B_{\rho}(0)}
$$

which together with (2), (4), and (8) yields

$$
\begin{aligned}
I(u) & =\frac{1}{b p^{2}} M_{u}^{p}-\int_{0}^{T} F(t, u(t)) d t-\frac{a^{p}}{b p^{2}} \\
& \geq \frac{a^{p-1}}{p}\|u\|_{E^{\alpha, p}}^{p}-\frac{(1-\varepsilon) a^{p-1}}{p C_{p}^{p}} \int_{0}^{T}|u(t)|^{p} d t
\end{aligned}
$$




$$
\begin{aligned}
& \geq \frac{a^{p-1}}{p}\|u\|_{E^{\alpha, p}}^{p}-\frac{(1-\varepsilon) a^{p-1}}{p}\|u\|_{E^{\alpha, p}}^{p} \\
& =\frac{\varepsilon a^{p-1}}{p}\|u\|_{E^{\alpha, p}}^{p}=\sigma, \quad \forall u \in \partial B_{\rho}(0) .
\end{aligned}
$$

Second, we claim that $I(\xi u) \rightarrow-\infty$ as $\xi \rightarrow \infty$. In fact, from $\left(\mathrm{H}_{3}\right)$, a simple argument can show that there are two constants $c_{1}, c_{2}>0$ such that

$$
F(t, x) \geq c_{1}|x|^{\mu}-c_{2}, \quad \forall(t, x) \in[0, T] \times \mathbb{R} .
$$

Thus, for each $u \in E_{0}^{\alpha, p} \backslash\{0\}, \xi \in \mathbb{R}^{+}$, we obtain from $\mu>p^{2}$ that

$$
\begin{aligned}
I(\xi u) & =\frac{1}{b p^{2}} M_{\xi u}^{p}-\int_{0}^{T} F(t, \xi u(t)) d t-\frac{a^{p}}{b p^{2}} \\
& \leq \frac{1}{b p^{2}} M_{\xi u}^{p}-c_{1} \int_{0}^{T}|\xi u(t)|^{\mu} d t+c_{2} T-\frac{a^{p}}{b p^{2}} \\
& =\frac{1}{b p^{2}}\left(a+b \xi^{p}\|u\|_{E^{\alpha, p}}^{p}\right)^{p}-c_{1} \xi^{\mu}\|u\|_{L^{\mu}}^{\mu}+c_{2} T-\frac{a^{p}}{b p^{2}} \\
& \rightarrow-\infty \quad \text { as } \xi \rightarrow \infty .
\end{aligned}
$$

Let

$$
g_{u}(s)=I(s u), \quad \forall s \in \mathbb{R}^{+} .
$$

Then, from what we have proved, $g_{u}$ has at least one maximum point $s(u)$ with maximum value greater than $\sigma>0$. Next, we prove that $g_{u}$ has a unique critical point for $s \in \mathbb{R}^{+}$, which then must be the global maximum point. Considering a critical point of $g_{u}$, one has

$$
\begin{aligned}
g_{u}^{\prime}(s) & =\left\langle I^{\prime}(s u), u\right\rangle \\
& =\|u\|_{E^{\alpha, p}}^{p} M_{s u}^{p-1} s^{p-1}-\int_{0}^{T} f(t, s u(t)) u(t) d t \\
& =0,
\end{aligned}
$$

which together with (5) yields

$$
\begin{aligned}
g_{u}^{\prime \prime}(s)= & b p(p-1)\|u\|_{E^{\alpha, p}}^{2 p} M_{s u}^{p-2} s^{2 p-2} \\
& +(p-1)\|u\|_{E^{\alpha, p}}^{p} M_{s u}^{p-1} s^{p-2}-\int_{0}^{T} f_{2}^{\prime}(t, s u(t)) u^{2}(t) d t \\
< & \|u\|_{E^{\alpha, p}}^{p} M_{s u}^{p-2}\left(b\left(p^{2}-1\right)\|u\|_{E^{\alpha, p}}^{p} s^{2 p-2}+a(p-1) s^{p-2}\right) \\
& -\frac{p^{2}-1}{s} \int_{0}^{T} f(t, s u(t)) u(t) d t \\
= & \|u\|_{E^{\alpha, p}}^{p} M_{s u}^{p-2}\left(b\left(p^{2}-1\right)\|u\|_{E^{\alpha, p}}^{p} s^{2 p-2}+a(p-1) s^{p-2}\right) \\
& -\left(p^{2}-1\right)\|u\|_{E^{\alpha, p}}^{p} M_{s u}^{p-1} s^{p-2} \\
= & a\|u\|_{E^{\alpha, p}}^{p} M_{s u}^{p-2}\left(p-p^{2}\right) s^{p-2} \leq 0 .
\end{aligned}
$$


Hence, if $s$ is a critical point of $g_{u}$, then it must be a strict local maximum point. This ensures the uniqueness of a critical point of $g_{u}$. Finally, from

$$
g_{u}^{\prime}(s)=\frac{1}{s}\left\langle I^{\prime}(s u), s u\right\rangle, \quad \forall t \in \mathbb{R}^{+},
$$

we obtain that, if $s$ is a critical point of $g_{u}$, then $s u \in \mathcal{N}$.

Let us define

$$
m=\inf _{\mathcal{N}} I
$$

Then we get from (7) that

$$
m \geq \inf _{\partial B_{\rho}(0)} I \geq \sigma>0
$$

Lemma 3.3 Assume that $\left(\mathrm{H}_{1}\right)-\left(\mathrm{H}_{3}\right)$ hold. Then there exists $u^{*} \in \mathcal{N}$ such that $I\left(u^{*}\right)=m$.

Proof By Lemma 2.9, we obtain that the functional

$$
u \rightarrow \int_{0}^{T} F(t, u(t)) d t, \quad \forall u \in E_{0}^{\alpha, p}
$$

is weakly continuous. Thus, as the sum of a convex continuous functional and a weakly continuous one, $I$ is weakly lower semi-continuous on $E_{0}^{\alpha, p}$.

Let $\left\{u_{k}\right\} \subset \mathcal{N}$ be a minimizing sequence of $I$, then one has

$$
I\left(u_{k}\right)=m+o(1), \quad G\left(u_{k}\right)=0 .
$$

Next, we prove that $\left\{u_{k}\right\}$ is bounded in $E_{0}^{\alpha, p}$. Based on the continuity of $\mu F(t, x)-x f(t, x)$ and $\left(\mathrm{H}_{3}\right)$, we see that there exists a constant $c>0$ such that

$$
F(t, x) \leq \frac{1}{\mu} x f(t, x)+c, \quad \forall(t, x) \in[0, T] \times \mathbb{R}
$$

Thus, from (11), we have

$$
\begin{aligned}
m+o(1) & =I\left(u_{k}\right) \\
& \geq \frac{1}{b p^{2}} M_{u_{k}}^{p}-\frac{1}{\mu} \int_{0}^{T} f\left(t, u_{k}(t)\right) u_{k}(t) d t-c T-\frac{a^{p}}{b p^{2}} \\
& =\frac{1}{b p^{2}} M_{u_{k}}^{p}-\frac{1}{\mu} M_{u_{k}}^{p-1}\left\|u_{k}\right\|_{E^{\alpha, p}}^{p}-c T-\frac{a^{p}}{b p^{2}} \\
& =M_{u_{k}}^{p-1}\left(\left(\frac{1}{p^{2}}-\frac{1}{\mu}\right)\left\|u_{k}\right\|_{E^{\alpha, p}}^{p}+\frac{a}{b p^{2}}\right)-c T-\frac{a^{p}}{b p^{2}} .
\end{aligned}
$$

Hence it follows from $\mu>p^{2}$ that $\left\{u_{k}\right\}$ is bounded in $E_{0}^{\alpha, p}$. 
Since $E_{0}^{\alpha, p}$ is a reflexive Banach space (see Lemma 2.6), up to a subsequence, we can assume $u_{k} \rightarrow u$ in $E_{0}^{\alpha, p}$. Moreover, from Lemma 2.9, one has $u_{k} \rightarrow u$ in $C([0, T], \mathbb{R})$. Next, we prove $u \neq 0$. By $\left(\mathrm{H}_{2}\right)$, we get that, for $\forall \varepsilon>0$, there exists a constant $\delta>0$ such that

$$
f(t, x) x \leq \varepsilon|x|^{p}, \quad \forall(t, x) \in[0, T] \times[-\delta, \delta] .
$$

Then, assume $\left\|u_{k}\right\|_{\infty} \leq \delta$, we obtain from (3), (4), and $u_{k} \in \mathcal{N}$ that

$$
\begin{aligned}
C_{\infty}^{-p}\left(a+b C_{\infty}^{-p}\left\|u_{k}\right\|_{\infty}^{p}\right)^{p-1}\left\|u_{k}\right\|_{\infty}^{p} & \leq\left(a+b\left\|u_{k}\right\|_{E^{\alpha, p}}^{p}\right)^{p-1}\left\|u_{k}\right\|_{E^{\alpha, p}}^{p} \\
& =\int_{0}^{T} f\left(t, u_{k}(t)\right) u_{k}(t) d t \\
& \leq \varepsilon \int_{0}^{T}\left|u_{k}(t)\right|^{p} d t \\
& \leq \varepsilon T\left\|u_{k}\right\|_{\infty}^{p}
\end{aligned}
$$

which is a contradiction from the arbitrariness of $\varepsilon$. Hence we have

$$
\|u\|_{\infty}=\underset{k \rightarrow \infty}{\lim }\left\|u_{k}\right\|_{\infty} \geq \delta>0
$$

and then $u \neq 0$. Thus, by Lemma 3.2, there exists $s \in \mathbb{R}^{+}$such that $s u \in \mathcal{N}$. Therefore, together with the fact that $I$ is weakly lower semi-continuous, we obtain

$$
m \leq I(s u) \leq \lim _{k \rightarrow \infty} I\left(s u_{k}\right) \leq \lim _{k \rightarrow \infty} I\left(s u_{k}\right)
$$

Finally, for $\forall u_{k} \in \mathcal{N}$, we see from (9) and (10) that $s=1$ is the global maximum point of $g_{u_{k}}$. So one has

$$
I\left(s u_{k}\right) \leq I\left(u_{k}\right),
$$

which together with (12) implies

$$
m \leq I(s u) \leq \lim _{k \rightarrow \infty} I\left(u_{k}\right)=m
$$

That is, $m$ is achieved at $s u \in \mathcal{N}$.

Now we give the proof of our main result.

Proof of Theorem 1.1 By Lemma 3.3, we get $u^{*} \in \mathcal{N}$ such that $I\left(u^{*}\right)=m=\inf _{\mathcal{N}} I>0$, that is, $u^{*}$ is a non-zero critical point of $\left.I\right|_{\mathcal{N}}$. Then, from Lemma 3.1, we know $I^{\prime}\left(u^{*}\right)=0$, and so $u^{*}$ is a nontrivial ground state solution of BVP (1).

\section{Acknowledgements}

The authors sincerely thank the editors and anonymous referees for the careful reading of the original manuscript and for valuable comments, which have improved the quality of our work. 
Availability of data and materials

Not applicable.

\section{Competing interests}

The authors declare that they have no competing interests.

\section{Authors' contributions}

The authors contributed equally in this article. They read and approved the final manuscript.

\section{Publisher's Note}

Springer Nature remains neutral with regard to jurisdictional claims in published maps and institutional affiliations.

Received: 18 October 2018 Accepted: 21 November 2018 Published online: 27 November 2018

\section{References}

1. Agarwal, R.P., O'Regan, D., Stanek, S.: Positive solutions for Dirichlet problems of singular nonlinear fractional differential equations. J. Math. Anal. Appl. 371, 57-68 (2010)

2. Arosio, A., Panizzi, S.: On the well-posedness of the Kirchhoff string. Trans. Am. Math. Soc. 348, 305-330 (1996)

3. Bai, Z., Lü, H.: Positive solutions for boundary value problem of nonlinear fractional differential equation. J. Math. Anal. Appl. 311, 495-505 (2005)

4. Benchohra, M., Hamani, S., Ntouyas, S.K.: Boundary value problems for differential equations with fractional order and nonlocal conditions. Nonlinear Anal. 71, 2391-2396 (2009)

5. Benson, D.A., Wheatcraft, S.W., Meerschaert, M.M.: The fractional-order governing equation of Lévy motion. Water Resour. Res. 36, 1413-1423 (2000)

6. Bernstein, S.: Sur une classe d'équations fonctionnelles aux déivés partielles. Bull. Acad. Sci. URSS Sér. Math. 4, 17-26 (1940)

7. Bisci, G.M., Repovs, D.: Higher nonlocal problems with bounded potential. J. Math. Anal. Appl. 420, 167-176 (2014)

8. Cavalcanti, M.M., Domingos Cavalcanti, V.N., Soriano, J.A.: Global existence and uniform decay rates for the Kirchhoff-Carrier equation with nonlinear dissipation. Adv. Differ. Equ. 6, 701-730 (2001)

9. Cresson, J.: Inverse problem of fractional calculus of variations for partial differential equations. Commun. Nonlinear Sci. Numer. Simul. 15, 987-996 (2010)

10. D'Ancona, P., Spagnolo, S.: Global solvability for the degenerate Kirchhoff equation with real analytic data. Invent. Math. 108, 247-262 (1992)

11. Darwish, M.A., Ntouyas, S.K.: On initial and boundary value problems for fractional order mixed type functional differential inclusions. Comput. Math. Appl. 59, 1253-1265 (2010)

12. Diethelm, K., Freed, A.D.: On the solution of nonlinear fractional order differential equations used in the modeling of viscoelasticity. In: Keil, F., Mackens, W., Voss, H., Werther, J. (eds.) Scientific Computing in Chemical Engineering I-Computational Fluid Dynamics, Reaction Engineering and Molecular Properties, pp. 217-224. Springer, Heidelberg (1999)

13. Ervin, V.J., Roop, J.P.: Variational formulation for the stationary fractional advection dispersion equation. Numer. Methods Partial Differ. Equ. 22, 558-576 (2006)

14. Fix, G.J., Roop, J.P.: Least squares finite-element solution of a fractional order two-point boundary value problem. Comput. Math. Appl. 48, 1017-1033 (2004)

15. Glockle, W.G., Nonnenmacher, T.F.: A fractional calculus approach of self-similar protein dynamics. Biophys. J. 68 46-53 (1995)

16. Hilfer, R.: Applications of Fractional Calculus in Physics. World Scientific, Singapore (2000)

17. Jiang, W.: The existence of solutions to boundary value problems of fractional differential equations at resonance. Nonlinear Anal. 74, 1987-1994 (2011)

18. Jiao, F., Zhou, Y.: Existence of solutions for a class of fractional boundary value problems via critical point theory. Comput. Math. Appl. 62, 1181-1199 (2011)

19. Jiao, F., Zhou, Y.: Existence results for fractional boundary value problem via critical point theory. Int. J. Bifurc. Chaos 22, Article ID $1250086(2012)$

20. Kilbas, A.A., Srivastava, H.M., Trujillo, J.J.: Theory and Applications of Fractional Differential Equations. Elsevier, Amsterdam (2006)

21. Kirchhoff, G.: Mechanik. Teubner, Leipzig (1883)

22. Kirchner, J.W., Feng, X., Neal, C.: Fractal stream chemistry and its implications for contaminant transport in catchments. Nature 403, 524-526 (2000)

23. Leibenson, L.S.: General problem of the movement of a compressible fluid in a porous medium. Izv. Akad. Nauk Kirg. SSSR 9, 7-10 (1983)

24. Lions, J.L.: On some questions in boundary value problems of mathematical physics. In: Contemporary Developments in Continuum Mechanics and Partial Differential Equations. Proc. Internat. Sympos., Inst. Mat., Univ. Fed. Rio de Janeiro, Rio de Janeiro, 1977. North-Holland Math. Stud., vol. 30, pp. 284-346. North-Holland, Amsterdam (1978)

25. Mainardi, F.: Fractional calculus: some basic problems in continuum and statistical mechanics. In: Carpinteri, A., Mainardi, F. (eds.) Fractals and Fractional Calculus in Continuum Mechanics, pp. 291-348. Springer, Wien (1997)

26. Pohozăev, S.I.: A certain class of quasilinear hyperbolic equations. Mat. Sb. 96, 152-166 (1975)

27. Samko, S.G., Kilbas, A.A., Marichev, O.I.: Fractional Integrals and Derivatives: Theory and Applications. Gordon \& Breach, New York (1993)

28. Zhang, Z., Yuan, R.: Infinitely-many solutions for subquadratic fractional Hamiltonian systems with potential changing sign. Adv. Nonlinear Anal. 4, 59-72 (2015) 